\title{
Computational Model of Unsteady Hydromechanics of Large Amplitude Gerstner Waves
}

\author{
Alexander Degtyarev ${ }^{1}$, Ivan Gankevich ${ }^{1, \star}$, Nataliia Kulabukhova ${ }^{1}$, and Vasily Khramushin ${ }^{1,2}$ \\ ${ }^{1}$ Saint Petersburg State University, Universitetskaya Emb. 7-9, 199034 St. Petersburg, Russia \\ ${ }^{2}$ Alexey Krylov All-Russian Scientific Shipbuilder Society, Saint Petersburg, Russia
}

\begin{abstract}
The computational experiments in the ship fluid mechanics involve the nonstationary interaction of a ship hull with wave surfaces that include the formation of vortices, surfaces of jet discontinuities, and discontinuities in the fluid under the influence of negative pressure. These physical phenomena occur not only near the ship hull, but also at a distance where the waves break as a result of the interference of the sea waves with waves reflected from the hull. In the study reported here we simulate the wave breaking and reflection near the ship hull. The problem reduces to determining the wave kinematics on the moving boundary of a ship hull and the free boundary of the computational domain. We build a grid of large particles having the form of a parallelepiped and, in the wave equation instead of the velocity field we integrate streams of fluid represented by functions as smooth as the wave surface elevation field. We assume that within the boundaries of the computational domain the waves do not disperse, i.e. their length and period stay the same. Under this assumption, we simulate trochoidal Gerstner waves of a particular period. This approach allows to simulate the wave breaking and reflection near the ship hull. The goal of the research is to develop a new method of taking the wave reflection into account in the ship motion simulations as an alternative to the classic method which uses added masses.
\end{abstract}

\section{Introduction}

The ship and sea wave surface motions do not involve an abundance of geometric forms and physical phenomena. The formation of waves due to the ship motion and the interaction between the ocean and the atmosphere are governed only by the continuity condition for a heavy fluid and the law of conservation of the energy.

A strict theoretical solution (and in fact the only solution) for large-amplitude wind waves at the surface of a heavy fluid was obtained in 1802 by Franz Josef von Gerstner [1]. The generic trochoidal wave mathematical model has large dispersion [2], the speed of the wave propagation depends on their length and period. As a result

- wave energy propagation speed becomes half the visible phase velocity of the wave crests,

- wave front constantly changes its phase, and

- waves are quantised into packets and the phenomena of wave transformation and propagation become nonstationary.

\footnotetext{
^e-mail: i.gankevich@spbu.ru
} 


\section{Computational model of intense sea waves}

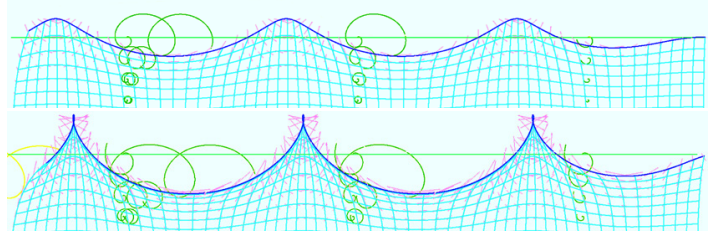

Figure 1. (Color online) Analytic solutions: progressive Gerstner wave (top), a wave of critical height, as a standing wave (bottom).

The Gerstner wave (Fig. 1) is a cycloid with the radius $r_{W}=1.134 \lambda_{W} h_{W} / 4 \pi$ of the particle trajectory being fixed relative to the flat wave surface level $z_{W}$, hence $z$-coordinates of the crest and trough are the same. Here $\lambda_{W}$ is the wave length, $h_{W}$ is the relative wave height defined on the interval $[0,1]$ with $h_{W}=1$ being the maximum wave height for which the crest does not break (Fig. 1). The vertical displacement of a fluid particle is given by

$$
\zeta_{Z}=r_{W} \cos x_{W} \exp \left(-2 \pi z_{W} / \lambda_{W}\right)
$$

The horizontal displacement of the same fluid particle with respect to its initial position for progressive wave is given by an analogous equation, but with a shift by one fourth of the phase:

$$
\zeta_{X}=-r_{W} \sin x_{W} \exp \left(-2 \pi z_{W} / \lambda_{W}\right) .
$$

The critical wave height of the Gerstner waves (Fig. 1) gives the correct ratio of the wave height to the wave length, but 60 degree slope limit for standing wave with steepness $\approx 1 / 4$ as well as 30 degree slope limit for progressive (traveling) wave with steepness $\approx 1 / 7$ are not correctly captured by the model.

The main focus of state of the art mathematical and computational models that simulate wave groups is on stochastic properties rather than fluid mechanics [3]. Our model is a modified version of the Gerstner wave which includes wave groups. They are described as a dependency between fluid particle trajectory radius and instantaneous displacement of the particle with respect to the calm sea level (Fig. 2).

We write the adjusted radius as $R_{W}^{A}=$ $K^{A} r_{W}\left(\cos x_{W}-1\right)$, where $K^{A} \in[1, \sqrt{2}]$ is the radius coefficient that makes the wave crests cnoidal and raises the mean sea level. We choose $K^{A}$ to be slightly less than 1 to reduce the effect of gusty winds on the wave form and to prevent the formation of cycloidal loops in the wave crests, that appear for waves with overly large amplitude, which may occur as a result of the interference with waves heading from the opposite direction.

The pressure on the windward slope of the wave is smaller, because the wind slides on the surface of the wave at a high speed makes the slope flatter, while on the leeward slope of the wave the wind speed drops significantly or even comes to nought and creates vorticity.

The coefficient of wind stress $K^{W}$ (the parameter that was shifted by one fourth of the phase) determines the asymmetry of steepness of windward and leeward slopes of the wave (Fig. 2): $R_{W}^{W}=$ $K^{W} r_{W}\left(\sin x_{W}-1\right)$, where $K^{W} \in[0,1]$. The coefficient is close to unity for fresh wind waves and 
close to nought for swell. For a two-dimensional sea surface, $K^{W}$ is used in dot product between the wind and wave direction vectors:

$$
\begin{aligned}
& \zeta_{Z}=r_{W} \cos x_{W} \exp \left(2 \pi\left[-z_{W}+r_{W} K^{A}\left(\cos x_{W}-1\right)+r_{W} K^{W} \sin x_{W}\right] / \lambda_{W}\right) \\
& \zeta_{X}=-r_{W} \sin x_{W} \exp \left(2 \pi\left[-z_{W}+r_{W} K^{A}\left(\cos x_{W}-1\right)+r_{W} K^{W} \sin x_{W}\right] / \lambda_{W}\right) .
\end{aligned}
$$

The energy conservation is defined by the Bernoulli's principle: $\rho V^{2} / 2+\rho g \zeta_{W}=$ const, where the particle velocity $V$ brings the largest contribution to balancing the pressure $\rho g \zeta_{W}$ on the wave surface down to nought for breaking waves.

\section{Trochoidal wave groups}

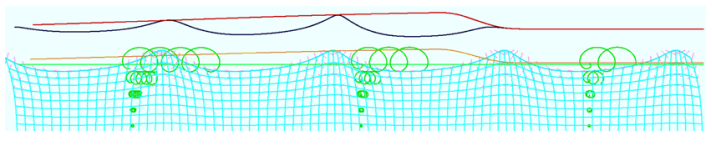

Figure 3. (Color online) Trochoidal wave groups.

In our modified model we simulate two wave surfaces simultaneously: one for regular waves with normal length and one for waves with nine times higher length, that propagate under the same laws but with half speed (Fig. 3). The product of these surfaces allows to simulate wave groups.

On the first entry the profile of the long wave is given by a specific smoothing function the form of which is close to the phase wave profile. This function defines continuous changes of the wave front phase which is needed to simulate waves produced by the ship.

There is also a simpler approach to simulate wave groups: a superposition of regular waves with slightly different periods propagating in opposite directions. The interference of waves of comparable lengths produces beats, in which the ninth wave has double height and is standing wave. This approach generally gives satisfactory wave surfaces, but does not work for waves produced by the ship, because these have intricate wave fronts.

\section{Direct numerical simulation of sea waves}

We use an explicit numerical scheme to simulate a sea wave surface that satisfies the continuity equation; we call it direct numerical simulation (Fig. 4). We use the following definitions for three sea wave systems, that are used in the scheme.

- Fresh wind waves have a period of 6-8 seconds near the shore and up to 10-12 seconds in the open ocean. The height of the wave is close to critical, that typically corresponds to 6 on the Beaufort scale with wave crests larger than 5-6 metres.

- Fresh swell waves skew from mean wind direction by $\approx 30$ degrees. When the storm in northern hemisphere increases, wind direction goes counterclockwise and vice versa, i.e. the swell is always present in the ocean. Swell waves are comparable to wind waves: their height is two times smaller than the critical wave height, and their length is 1.5-2 times larger. 
- Old swell waves are long waves that come from higher latitudes. Their height is two times smaller than than of the wind waves and fresh swell, their length is two times larger, and their direction is close to meridional (i.e. south in northern latitudes and vice versa ${ }^{1}$ ).

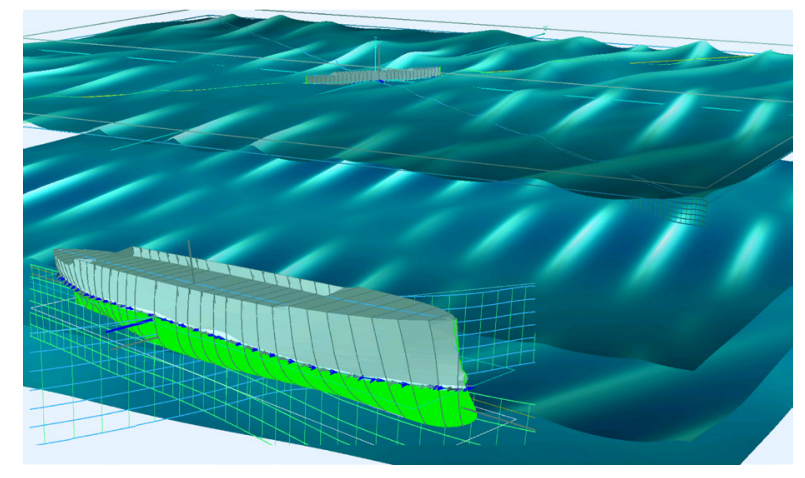

Figure 5. (Color online) In the course of the simulation we visualise all three wave systems and create a view of ship hull dynamics and sea wave profiles in a different convenient scale.

These waves may add up in unfavourable way to a wave with the height of 13-15 metres, however, in real world mean wave height will be $8-10$ metres. Wave groups have the ninth wave with double height, breaking crest and wave slope larger than 45 degrees.

Oceanographers use well-established solutions [4] for regular progressive waves of arbitrary shape. Using trochoidal waves as a source, we fix wave periods and speeds in time to satisfy continuity equation and energy conservation law. We simulate all three wave systems (described above) simultaneously and indepedently (Fig. 5) and add individual wave surfaces together to produce the resulting wave surface.

\section{Conclusion}

We use explicit numerical schemes to simulate a modified version of the Gerstner waves. We simulate particle drift in the upper fluid layers by changing the curvature of the trajectory depending on the instantaneous change of the wave surface elevation. Our model is nonstationary, hence ship motions can also be nonstationary. The computational power of a desktop computer is enough for performing such simulations in real-time, and these types of simulations can even be performed on the board of the ship to chose efficiently the optimal mode of ship operation.

\section{Acknowledgement}

Research work is supported by Saint Petersburg State University (grant no. 26520170 and 39417213).

\section{References}

[1] F. Gerstner, Theorie der wellen, Annalen der Physik 32, 412 (1809), republished from an 1802 paper.

[2] A. Sommerfeld, Mechanik der deformierbaren Medien, Vorlesungen über theoretische Physik (Akademische Verlagsgesellschaft Becker \& Erler, 1945)

[3] P.A. Anastopoulos, K.J. Spyrou, C.C. Bassler, V. Belenky, Towards an improved critical wave groups method for the probabilistic assessment of large ship motions in irregular seas, Probabilistic Engineering Mechanics 44, 18 (2016), special Issue Based on Papers Presented at the 7th International Conference on Computational Stochastic Mechanics (CSM7)

[4] A. Poplavskii, V. Khramushin, K. Nepop, Y. Korolev, Operational forecast of the tsunami on the sea shores of the Far East of Russia (Yuzhno-Sakhalinsk: Institute of Marine Geology and Geophysics Far Eastern Branch Russian Academy of Sciences, 1997), in Russian

\footnotetext{
${ }^{1}$ The wind blows into the compass rose, the waves propagate in the direction of the rose.
} 